\title{
Impact of the South African Mental Health Care Act No. 17 of 2002 on regional and district hospitals designated for mental health care in KwaZulu- Natal
}

\author{
Suvira Ramlall, Jennifer Chipps, Maurice Mars
}

Background. The South African Mental Health Care Act (the Act) No. 17 of 2002 stipulated that regional and district hospitals be designated to admit, observe and treat mental health care users (MHCUs) for 72 hours before they are transferred to a psychiatric hospital.

Methods. Medical managers in 49 'designated' hospitals in KwaZulu-Natal (KZN) were surveyed on infrastructure, staffing, administrative requirements and mental health care user case load pertaining to the Act for the month of July 2009.

Results. Thirty-six (73.4\%) hospitals responded to the survey; $30(83.3 \%)$ stated that the Act improved mental health care for MHCUs through the protection of their rights, provision of least restrictive care, and reduction of discrimination; $10(27.8 \%)$ had a psychiatric unit and, of the remaining 26 hospitals, 11 (30.6\%) had general ward beds dedicated for psychiatric admissions;
16 (44.4\%) had some form of seclusion facility; and 24 (66.7\%) provided an outpatient psychiatric service. Seventy-six per cent of admissions were involuntary or assisted. Thirteen of the $32(40.6 \%)$ state psychiatrists in KZN were employed at 8 of these hospitals. Designated hospitals expressed dissatisfaction with the substantial administrative load required by the Act. The Review Board had not visited 29 (80.6\%) hospitals in the preceding 6 months.

Conclusion. Although 'designated' hospitals admit and treat assisted and involuntary MHCUs, they do so against a backdrop of inadequate infrastructure and staff, a high administrative load, and a low level of contact with Review Boards.

S Afr Med J 2010; 100: 667-670.
'Mental health is an integral and essential component of health.' Neuropsychiatric disorders contribute significantly to disability and health care cost in society, ${ }^{2}$ and rank third in their contribution to burden of disease in SA. ${ }^{3}$ To ensure adequate access and treatment for mental health care users (MHCUs), human, social and financial resources are necessary. ${ }^{4}$ Internationally, 32\% of 191 countries surveyed did not have a specified budget for mental health, ${ }^{5}$ and $36 \%$ of countries spent less than $1 \%$ of their total health budgets on mental health. ${ }^{6}$ Scarce resources, inequity of distribution and inefficiency of resource use characterise mental health services in low- and middleincome countries. ${ }^{1}$ Mental health was a low priority on South Africa's public health agenda, the lack of an action plan being one of the shortcomings. ${ }^{7}$

Historically, mental health services in KwaZulu-Natal (KZN) had been centred on a few large mental hospitals and stand-alone clinics. The Mental Health Care Act No. 17 of 2002 (the Act) ${ }^{8}$ introduced radical changes. Selected regional and district public hospitals were designated under the Act to perform 72-hour observations on involuntary and assisted MHCUs. These 'designated hospitals' (DHs) would ensure increased accessibility and availability of mental health care services locally and reduce the need for premature or unnecessary transfers to psychiatric hospitals, as well as allowing screening for medical conditions presenting as psychiatric disorders,

Department of Psychiatry, Nelson R Mandela School of Medicine, University of KwaZulu-Natal, Durban

Suvira Ramlall, MB ChB, FC Psych (SA)

Department of TeleHealth, Nelson R Mandela School of Medicine, University of KwaZulu-Natal, Durban

Jennifer Chipps, BSc (Nursing), BSc (Psychology), MPH (Univ NSW) Maurice Mars, MB ChB, MD which could be as high as $24.2 \%{ }^{9} \mathrm{KZN}$ has 8 specialist psychiatric and 63 district and regional hospitals; $50(70.4 \%)$ of the district and regional hospitals have been designated to provide mental health services and admit involuntary and assisted MHCUs for 72-hour observations.

Local stakeholders have raised concerns that this 'designation' has not been accompanied by a dedicated budget, implementation plan or increase in staff and facilities. Petersen expressed concern that de-institutionalisation and comprehensive integrated mental health care in South Africa were hampered by a lack of resources for mental health care as well as the inefficient use of existing mental health resources. ${ }^{10}$ In $\mathrm{KZN}, 0.03 \%$ of the total health budget is spent on mental health, a figure that has not increased in the last decade (personal communication, KZN Department of Health). Local psychiatrists in South Africa have raised concerns on implications of the Act, specifically on the requirement for 72-hour observation and admission. ${ }^{11,12}$ Moosa and Jeena feared that the implementation of involuntary treatment within a health care service that was plagued by human resource and infrastructure constraints could threaten the very rights of patients that the Act sought to uphold. ${ }^{13}$

Another major change in the Act was the appointment of Mental Health Review Boards. These boards are 'quasi-judicial authorities, ${ }^{14}$ tasked to uphold the human and health rights of people with mental illness and intellectual disability. Their role is to assist in promoting and protecting international, regional and nationally determined human rights of people with mental disorders and intellectual disability. ${ }^{14}$ The first Review Board appointments in KZN were beset with delays in the appointment process and provision of offices and administrative support, which frustrated the functioning of these boards. In addition, the Act stated that 'A Review Board may determine its own procedures for conducting business'. In KZN, prior to the publication of a standard operating procedure manual in 2007, ${ }^{14}$ the roles and responsibilities of the Review Board were unclear. The term of office of the first set of appointed boards came to an end in August 2009.

There has been no formal survey of the impact of the Act on the designated hospitals. An audit of the Act conducted in 2002 in the 
Western Cape revealed that many general hospitals were unable to provide mental health data, ${ }^{15}$ but the findings were not published owing to a low response rate (personal communication). We aim to describe the influence of the Act on designated hospitals in KZN in terms of: (i) caseload; (ii) infrastructure and staffing to provide mental health care services; (iii) administrative load of the Act; (iv) the level of relevant training received by staff; and $(v)$ the role of Review Boards.

\section{Methods}

A quantitative survey of the medical managers of 50 designated hospitals in KZN was conducted for a randomly selected month (July 2009). Psychiatric hospitals and psychiatric chronic care facilities $(N=8)$ were excluded. One designated hospital was also excluded as it has a psychiatric hospital attached to it $(N=49)$. Participation was voluntary, and medical managers of designated hospitals were requested to complete a questionnaire that assessed the capacity of their hospitals to provide mental health services and the administrative impact of the Act. Opinion was sought on helpful activities that could assist the hospitals to improve service delivery. The content of the questionnaire was based on South African and international indicators and norms for mental health services. ${ }^{15-24}$ The study received ethical clearance from the Human and Social Sciences Research and Ethics Committee of the University of KwaZulu-Natal and the Research Committee of the Department of Health in KZN. All data were treated as confidential, and no individual district or hospital has been identified.

The study yielded quantitative and qualitative data. Quantitative data were analysed using the Statistical Package for Social Sciences (SPSS) version 15.0 (2006). Qualitative data were coded according to commonalities. Estimates include 95\% confidence intervals (95\% CI) and associations were tested using two-sided Fisher's exact tests. The capacity to provide services was measured in terms of the MHCU caseload on these hospitals, the availability of appropriate physical infrastructure, and the presence of staff trained in psychiatry. MHCU caseload was measured through MHCU attendances in psychiatric outpatient services and MHCU admissions to the designated hospitals. Support was measured in terms of the availability of staff trained in psychiatry, perceptions of adequacy of support received, and relevant training of medical and nursing staff.

\section{Results}

Thirty-six (74\%) of the 49 hospitals responded - 9 regional and 27 district hospitals. Hospitals varied in size from 245 to 1200 beds (median 453 beds) and from 80 to 531 beds (median 212) for regional and district hospitals, respectively. All percentages are based on the 36 responding hospitals.

Thirty (83.3\%) hospitals stated that the Act improved mental health care for MHCUs by protecting their rights, providing least restrictive care, and reducing discrimination. Of these 30 hospitals, 7 (23.3\%) were regional, 23 (76.7\%) had no psychiatric unit, and 25 (83.3\%) had no resident psychiatrist.

\section{Psychiatric inpatient facilities Inpatient facilities}

Twenty-three (63.9\%) hospitals reported that they did not have appropriate or adequate facilities to provide psychiatric services as required by the Act. Five of the 23 were regional hospitals, of which 3 had resident psychiatrists. Common complaints related to the lack of sufficient beds, staff and seclusion rooms to accommodate the clinical demand, and the challenges of managing disruptive patients in a general hospital setting. MHCUs were either admitted to a psychiatric unit or a bed in a general ward.

Fifteen hospitals (41.7\%) admitted MHCUs to general medical or surgical wards; $10(27.8 \%)$ had a psychiatric unit in the hospital and 11 (30.6\%) reported having beds designated for MHCU admissions. One psychiatric unit was vacant as funds to commission it were unavailable. Four hospitals with psychiatric units had additional beds designated for MHCUs within general surgical and/or medical wards to cater for overflow admissions or the opposite gender. The 21 hospitals with units and/or designated beds provided a total of 289 beds for admission of acute MHCUs in the month of July 2009. Of the 289 beds, 207 (71.6\%) beds were in the 10 hospitals with psychiatric units, (181 in the units and 26 in general wards in 4 of the hospitals).

\section{Seclusion facilities}

Sixteen (44.4\%) hospitals reported having some form of seclusion facility: 6 of the 10 hospitals (60\%) with psychiatric units and 7 of the 11 hospitals $(63.6 \%)$ with dedicated psychiatric beds. Three of the $15(20 \%)$ hospitals with no dedicated beds reported having access to a seclusion facility. Those with seclusion facilities reported dissatisfaction with the infrastructure or the number of facilities. In 5 hospitals, existing wards were inadequately refurbished seclusion facilities or medical isolation wards were doubling up as 'seclusion' facilities.

\section{Psychiatrists based at designated hospitals}

Twenty-five (69.4\%) hospitals reported that they did not have the necessary medical and/or nursing staff to provide required services. Six of the 25 hospitals (24\%) were regional hospitals, 3 of which had units and 4 of which had resident psychiatrists. At the time of the study, 13 (40.6\%) of a total of 32 state psychiatrists were resident at the designated hospitals: 12 at 7 hospitals with psychiatric units and 1 in a regional hospital with no unit.

\section{MHCU caseload}

\section{Outpatients}

Twenty-four (66.7\%) hospitals, of which 6 had resident psychiatrists, reported providing a dedicated psychiatric outpatient service. Of the 20 hospitals that provided data on outpatient services, a total of 8341 MHCUs attended in July - an average of 417 (range 184.6 - 649.5) attendances per hospital (median 277).

\section{Inpatients}

The profile of admissions is shown in Table I.

\section{Administrative load of the Act}

Three hospitals $(8.3 \%)$ did not have the necessary admission forms available, of which 2 reported admitting involuntary or assisted MHCUs in July. Staff at 24 hospitals (66.7\%) were perceived to be reasonably proficient in completing MHCA forms. Twenty-six (72.2\%) hospitals had a Commissioner of Oaths available in the hospital to authenticate mental health applications under the Act; $16(44.4 \%)$ reported that forms were not being forwarded to the Review Boards; and $12.5 \%$ of hospitals had psychiatrists compared with $62.5 \%(15 / 24)$ without psychiatrists $(p=0.04)$. A major concern was that the administrative burden of the Act was considerable. Comments ranged from the large number of forms to complete, to the strictly defined time-frames within which these had to be completed and forwarded to the various structures, e.g. 'Too much paperwork.' 'Too much paperwork leads to confusion and frustration.' 'Staff do 
Table I. MHCUs admitted by type of facility (July 2009)

\begin{tabular}{|c|c|c|c|}
\hline $\begin{array}{l}\text { Type of psychiatric facility in } \\
\text { hospital }\end{array}$ & $\begin{array}{l}\text { Total number of MHCU } \\
\text { admissions }\end{array}$ & $\begin{array}{l}\text { Involuntary and assisted } \\
\text { admissions (\% of total MHCU } \\
\text { admissions) }\end{array}$ & Median admissions per hospital \\
\hline Psychiatric unit only (6) & 190 & $169(88.9 \%)$ & 33 \\
\hline $\begin{array}{l}\text { Psychiatric unit and designated } \\
\text { beds ( } 4 \text { ) }\end{array}$ & 273 & $144(52.7 \%)$ & 61.5 \\
\hline Designated beds only (11) & 187 & $154(82 \%)$ & 9 \\
\hline No designated beds (15) & 99 & $99(100 \%)$ & 4 \\
\hline DHs $(N=36)$ & 749 & $566(75.6 \%)$ & 8.5 \\
\hline
\end{tabular}

not have time to fill forms in.' Additional concerns were a lack of dedicated administrative personnel, and insufficient photocopying machines to ensure copies were sent timeously to managers, MHCUs, families and review boards.

\section{Staff training and support}

Eleven $(30.6 \%)$ of the hospitals stated that their staff had received adequate support to provide 72-hour observation. Hospitals with psychiatric units and those without were significantly different; $60 \%(6 / 10)$ of hospitals with psychiatric units agreed that they had adequate support, compared with $19.2 \%$ (5/26) of hospitals with no units $(p=0.04)$.

Twenty (55.6\%) hospitals agreed that they had received adequate training in the Act, specifically those with psychiatrists $(87.5 \% \mathrm{v}$. $46.4 \%, p=0.05)$ and hospitals with psychiatric units (90\% v. $42.3 \%$, $p=0.02)$. Training in the Act was received by nurses at $26(72.2 \%)$ and doctors at $19(52.8 \%)$ hospitals, and training in the management of MHCUs by nurses and doctors at 25 (69.4\%) and 15 (41.7\%) hospitals respectively. Of supportive activities, training was identified as potentially useful.

\section{Review Boards}

Reported visits by the Review Board were infrequent; 7 (19.4\%) hospitals reported visits in the preceding 6 months, and 10 had either never been visited or were last visited more than 2 years before the survey. Four hospitals reported that when visits did occur, they were useful, with the Review Board providing guidance and management on the care of MHCUs.

The experience of most hospitals with the Review Board was unsatisfactory. Comments included: 'No Review Board exists', 'Is noticeable by its absence', 'Difficult to communicate with Review Board, 'Not very useful' and 'Review Board is very critical rather than helpful, and visits only occurred on weekends and public holidays'.

Even when visits did occur, it was felt that their usefulness was limited: 'To date, the visits by the Review Board has served no constructive or useful purpose. 'Usefulness is hindered by their inability to compel the Minister of Health to provide dedicated psychiatric structure and staff establishment for psychiatry'. The 'main problem is a lack of beds which the Review Board has no control over'. Another major concern expressed was the response lag in receiving final Review Board authorisation and High Court approval, questioning the capacity of the Review Board or judiciary to intervene timeously in the event of a violation of the Act.

\section{Discussion}

Five years since the implementation of the Act, integration of mental health services into the district health system appears to have been facilitated, but concerns remain about the capacity of these hospitals to meet the requirements of the Act. This study highlights the hiatus between the intention of the Act and the capacity of designated hospitals to meet the legislative requirements.

\section{Infrastructure}

One in 5 people is estimated to suffer some form of mental illness. ${ }^{4}$ With only $66.7 \%$ of designated hospitals offering outpatient services, their effectiveness in addressing the burden of mental illness must be questioned. In addition, designated hospitals admitted over $75 \%$ of the MHCUs as involuntary or assisted patients, catering almost exclusively for the most severe of the mentally ill population. This fact was also highlighted in a KZN rural district site where it was found 'that the decentralization process in Primary Health Care Clinics remains largely limited to emergency management of psychiatric patients and ongoing psychopharmacological care of patients with stabilized chronic conditions. ${ }^{10}$

In such conditions, the shortage of specialist facilities, admission beds and staff trained in psychiatry is of concern. Even though $75.6 \%$ of psychiatric admissions to the 36 designated hospitals were involuntary or assisted, only 10 hospitals reported having a psychiatric unit. The availability of dedicated resources, such as a psychiatric unit, positively influenced admission rates, the provision of outpatient services and the appointment of appropriately trained psychiatric staff. The characteristics of the physical environment in which MHCUs receive care affect their outcomes and satisfaction, safety, staff satisfaction, staff efficiency and organisational outcomes. ${ }^{25}$

With 15 (41.6\%) hospitals having no dedicated beds at all for psychiatric patients, and only $44.4 \%$ of all the hospitals having dedicated facilities for seclusion, their admission of acutely disturbed MHCUs represents a potential hazard and increases the risk of violent and destructive incidents that will perpetuate the misconception that all MHCUs are dangerous. ${ }^{26}$

While 7 of the 10 hospitals with psychiatric units had dedicated psychiatrists, the others relied on staff from general medical wards with minimum experience of handling MHCUs. Capacity must be developed in health personnel at all establishments to manage MHCUs effectively and safely. Nearly half of the hospitals ranked training in the Act and management of MHCUs as potentially helpful. In a climate of significant shortages in clinical staff, a critical review of the administrative burden of the Act must also be undertaken, without compromising the rights of MHCUs or the strengths of the Act.

\section{Administration of the Act}

'The Act goes a long way towards improving the human rights of people with mental illness and intellectual disability; however, it is 
only in the successful implementation of the law, including proficient Review Boards, that these rights will become concrete reality for people. ${ }^{14}$ Review Boards should guide and support the hospitals and protect the rights of MHCUs. ${ }^{8}$ Although the Act does not stipulate that Board members have to visit hospitals, the procedure manual states: 'Review Board members may consider it necessary to visit health establishments acting as "protectors" of the rights of people who have been committed ... This may involve investigating conditions at facilities ....14 The low frequency of Board visits to hospitals in the first 6 months of the year raises concerns. Firstly, although paper reviews are permitted by the Act, this implies that decisions of the medical staff at these hospitals were accepted by the Board without investigation, and that these hospitals lodged no complaints or appeals. Secondly, the sub-optimum (qualitatively and quantitatively) inpatient facilities reported by the majority of hospitals have not invoked the concern of the Review Boards in these areas, even though they are tasked with investigating 'abuse, neglect and exploitation' of MHCUs. ${ }^{8}$ Thirdly, the Review Board is strategically placed between consumers and clinicians, and the Ministry of Health and the judiciary, to advocate mental health. The low level of interaction with designated hospitals suggests that this opportunity has not been exploited. Designated to perform a 'critical and legally-specified role, ${ }^{14}$ the Boards must function optimally and be available and accessible at all times to MHCUs, mental health care practitioners and the public.

\section{Conclusion}

Even though the presence of mental health policies does not ensure resource allocation, policies should initiate a process of change. ${ }^{5}$ The Act appears to have improved access to mental health care services in the province, but the study suggests that significant gaps in infrastructure, staffing, training and administrative requirements still must be addressed before implementation can be deemed a success.

\section{References}

1. Saxena S, Thornicroft G, Knapp M, Whiteford H. Resources for mental health: scarcity, inequity, and inefficiency. Lancet 2007;370:878-889.

2. WHO. The Global Burden of Disease: 2004 Update. Geneva: World Health Organization, 2004.

3. Bradshaw D, Norman R, Schneider M. A clarion call for action based on refined DALY estimates for South Africa. Editorial. S Afr Med J 2007;97:438-440.

4. WHO. The World Health Report 2001 - Mental Health: New Understanding, New Hope. Geneva: World Health Organization, 2001.

5. Saxena S, Sharan P, Saraceno B. Budget and financing of mental health services: baseline information on 89 countries from WHO's project atlas. J Ment Health Policy Econ 2003; 6(3): 135-143.

6. WHO. Mental Health Atlas. Geneva: World Health Organization, 2005.

7. The Mental Health and Poverty Project. Policy Brief 3. The Current State of Mental Health Policy in South Africa. Cape Town: Mental Health and Poverty Project, University of Cape Town, 2008
8. Mental Health Care Act, No. 17 (2002). Government Gazette. Pretoria: Government Printing Works, 2002.

9. Saloojee S. Routine pre-admission laboratory screening investigations in aggressive patients who require sedation in the emergency department necessary or unnecessary. South African Journal of Psychiatry 2009;15:67-71.

10. Petersen I, Bhana A, Campbell-Hall V, et al. Planning for district mental health services in South Africa: a situational analysis of a rural district site. Health Policy Plan 2009;24(2):140-150.

11. Szabo CP. The Mental Health Care Act: challenges and opportunities. South African Psychiatric Review 2006;9:1-5.

12. Burns JK. Implementation of the Mental Health Care Act (2002) at district hospitals in South Africa: Translating principles into practice. S Afr Med J 2008; 98(1):46-51.

13. Moosa MYH, Jeena FY. Involuntary treatment of psychiatric patients in South Africa. African Journal of Psychiatry 2008; 11:109-112.

14. Mental Health Review Board Orientation Guideline and Training Manual. Pretoria: South African Department of Health, 2007.

15. Lund C, Kleintjes S, Kakuma R, Flisher AJ. Public sector mental health systems in South Africa: inter-provincial comparisons and policy implications. Soc Psychiatry Psychiatr Epidemiol 2010; 45(3):393-404.

16. Lund C, Flisher AJ. South African Mental Health Process Indicators. J Ment Health Policy Econ 2001;4(1):9-16.

17. Lund C, Flisher AJ. Staff/bed and staff/patient ratios in South African public sector mental health services. S Afr Med J 2002;92(2):157-161.

18. Lund C, Flisher AJ. Staff/population ratios in South African public sector mental health services. S Afr Med J 2002; 92(2):161-164.

19. Lund C, Flisher AJ. Community/hospital indicators in South African public sector mental health services. J Ment Health Policy Econ 2003; 6(4):181-187.

20. Lund C, Flisher AJ, Lee T, Porteus K, Robertson BA. A model for estimating mental health service needs in South Africa. S Afr Med J 2000;90(10):10191024.

21. Lund C, Flisher AJ, Porteus K, Lee T. Bed/population ratios in South African public sector mental health services. Soc Psychiatry Psychiatr Epidemiol 2002; 37(7):346-349.

22. Lund C, Stein DJ, Flisher AJ, Mehtar S. Challenges faced by South African health services in implementing the Mental Health Care Act. S Afr Med J 2007;97(5): 352-353.

23. McEwan K, Goldner EM. Accountability and Performance Indicatorsfor Mental Health Services and Supports: A Resource Kit. Vancouver: Department of Psychiatry, University of British Columbia, 2001.

24. Muller L, Flisher AJ. Standards for mental health services in South Africa. The South African Psychiatry Review 2006;9(1a):1-40.

25. Smith R, Watkins N. Therapeutic environments. Washington DC: Academy of Architecture for Health, 2008.

26. Saville S. Petrol junkie: Psychiatric patient runs amok damaging cars at Northdale Hospital. Natal Witness 20 February 2010.

Accepted 16 July 2010 